\title{
PROPERLY EMBEDDED LEAST AREA PLANES IN GROMOV HYPERBOLIC 3-SPACES
}

\author{
BARIS COSKUNUZER
}

(Communicated by Alexander N. Dranishnikov)

\begin{abstract}
Let $X$ be a Gromov hyperbolic 3-space with cocompact metric, and $S_{\infty}^{2}(X)$ the sphere at infinity of $X$. We show that for any simple closed curve $\Gamma$ in $S_{\infty}^{2}(X)$, there exists a properly embedded least area plane in $X$ spanning $\Gamma$. This gives a positive answer to Gabai's conjecture from 1997. Soma has already proven this conjecture in 2004. Our technique here is simpler and more general, and it can be applied to many similar settings.
\end{abstract}

\section{INTRODUCTION}

Let $X$ be a Gromov hyperbolic 3-space with cocompact metric, and $S_{\infty}^{2}(X)$ the sphere at infinity of $X$. Let $\Gamma$ be a given simple closed curve in $S_{\infty}^{2}(X)$. The asymptotic Plateau problem asks the existence of a least area plane $\Sigma$ in $X$ with asymptotic boundary $\Gamma$, i.e. $\partial_{\infty} \Sigma=\Gamma$. The author gave a positive answer to this question, and showed the existence of such least area planes asymptotic to given curves in $S_{\infty}^{2}(X)$ in [Co].

Even though we know the existence of such least area planes in $X$ asymptotic to given curves in $S_{\infty}^{2}(X)$, proper embeddedness of these least area planes is also another interesting question came out in Gabai's renowned paper [Ga1]. In that paper, he solved the asymptotic Plateau problem for hyperbolic 3-spaces with a cocompact metric, and he showed that, for any simple closed curve in $S_{\infty}^{2}(\widetilde{M})$, there exists a least area plane spanning the given curve in $\widetilde{M}$ by using laminations where $\widetilde{M}$ is the universal cover of a closed hyperbolic manifold $M$ with any Riemannian metric. Also, he conjectured that there exists a properly embedded least area plane in $\widetilde{M}$ for a given simple closed curve in $S_{\infty}^{2}(\widetilde{M})$, [Ga1, 3.12].

On the other hand, there is no known example of a nonproperly embedded least area plane in $\mathbb{H}^{3}$ (or any Gromov hyperbolic 3-space with cocompact metric) spanning a simple closed curve in $S_{\infty}^{2}\left(\mathbb{H}^{3}\right)$. There is the construction of a nonproper least area plane in $\mathbb{H}^{3}$ by Freedman and He communicated to Gabai [Ga2]. It is not clear to the author as to how a plane constructed in this manner can have a limit set as a simple closed curve.

Teruhiko Soma gave a positive answer to Gabai's conjecture on hyperbolic 3space with cocompact metric in [So1]. Then, he generalized his result to Gromov hyperbolic 3-spaces with cocompact metric in [So2]. In this paper, we will show

Received by the editors October 16, 2006 and, in revised form, February 2, 2007.

2000 Mathematics Subject Classification. Primary 53A10; Secondary 57M50.

The author was supported by NSF Grant DMS-0603532.

(c)2007 American Mathematical Society 1427

Reverts to public domain 28 years from publication 
the same results with a simpler and more general technique which is applicable to many different settings. Our main result is the following:

Theorem 3.3. Let $X$ be a Gromov hyperbolic 3-space with cocompact metric, and $S_{\infty}^{2}(X)$ be the sphere at infinity of $X$. Let $\Gamma$ be a given simple closed curve in $S_{\infty}^{2}(X)$. Then, there exists a properly embedded least area plane $\Sigma$ in $X$ with $\partial_{\infty} \Sigma=$ $\Gamma$.

A short outline of the proof is as follows. First, we consider the sequence of least area planes $\left\{\Sigma_{i}\right\}$ in $X$ with $\partial_{\infty} \Sigma_{i}=\Gamma_{i}$ such that $\Gamma_{i} \rightarrow \Gamma$ in $S_{\infty}^{2}(X)$ from one side. Then, we define a sequence of least area disks $\left\{D_{i}\right\}$ such that $D_{i} \subset \Sigma_{i}$ and $\partial D_{i} \rightarrow \Gamma$. By using the results in [Co], we show that $\left\{D_{i}\right\}$ converges to a nonempty lamination $\sigma$ by least area planes with $\partial_{\infty} \sigma=\Gamma$. Then, by using the fact that the least area disks stay in one side of the lamination, we prove that this lamination contains only one least area plane $\mathcal{P}$, i.e. $\sigma=\mathcal{P}$. Since $\sigma$ is a closed subset of $X$, $\sigma=\mathcal{P}$ must be a properly embedded least area plane with $\partial_{\infty} \mathcal{P}=\Gamma$.

\section{Preliminaries}

A 3-manifold $M$ is called a Gromov hyperbolic manifold if its fundamental group $\pi_{1}(M)$ is a word hyperbolic (or Gromov hyperbolic) group [Gr]. We call $X$ a Gromov hyperbolic 3-space with cocompact metric if $X$ is the universal cover of a Riemannian closed orientable irreducible Gromov hyperbolic 3-manifold $M$ where the metric on $X$ is induced from that on $M$. By [BM], $X$ is homeomorphic to an open ball in $\mathbb{R}^{3}$. Since $X$ is a Gromov hyperbolic 3 -space, it has a natural compactification $\bar{M}$ where $\bar{M}=M \cup \partial_{\infty} M$. Here, $\partial_{\infty} M$ is the sphere at infinity $S_{\infty}^{2}(X)$, and a point on $S_{\infty}^{2}(X)$ corresponds to an equivalence class of infinite rays in $X$ where two rays are equivalent if they are asymptotic. A hyperbolic 3-space with cocompact metric is the universal cover of a closed orientable irreducible hyperbolic 3-manifold $M$ with any Riemannian metric $\rho$ on $M$. Hyperbolic 3-spaces with cocompact metric (in particular $\mathbb{H}^{3}$ ) are special cases of Gromov hyperbolic 3spaces with cocompact metric.

We call a disk $D$ a least area disk if $D$ has the smallest area among the disks with the same boundary $\partial D$. A plane is a subset of $X$ with the topological type of an open disk, and complete with the induced path metric from $X$. We call a plane $P$ a least area plane if any subdisk in the plane $P$ is a least area disk. A plane is properly embedded if the preimage of any compact set is compact in the plane.

A codimension-1 lamination $\sigma$ in $X$ is a foliation of a closed subset of $X$ with 2-manifolds (leaves) such that $X$ is covered by charts of the form $I^{2} \times I$ where a leave passes through a chart in a slice of the form $I^{2} \times\{p\}$ for $p \in I$. Here and later, we abuse notation by also letting $\sigma$ denote the underlying space of its lamination.

The sequence $\left\{D_{i}\right\}$ of embedded disks in a Riemannian manifold $X$ converges to the lamination $\sigma$ if

i) For any convergent sequence $\left\{x_{n_{i}}\right\}$ in $X$ with $x_{n_{i}} \in D_{n_{i}}$, where $n_{i}$ is a strictly increasing sequence, $\lim x_{n_{i}} \in \sigma$.

ii) For any $x \in \sigma$, there exists a sequence $\left\{x_{i}\right\}$ with $x_{i} \in D_{i}$ and $\lim x_{i}=x$ such that there exist embeddings $f_{i}: D^{2} \rightarrow D_{i}$ which converge in the $C^{\infty}$-topology to a smooth embedding $f: D^{2} \rightarrow L_{x}$, where $x_{i} \in f_{i}\left(\operatorname{Int}\left(D^{2}\right)\right)$, and $L_{x}$ is the leaf of $\sigma$ through $x$, and $x \in f\left(\operatorname{Int}\left(D^{2}\right)\right)$.

We call such a lamination $\sigma$ a $D^{2}$-limit lamination. 
Theorem 2.1 ([Co]). Let $\Gamma$ be a simple closed curve in $S_{\infty}^{2}(X)$ where $X$ is a Gromov hyperbolic 3-space with cocompact metric. Then there exists a $D^{2}$-limit lamination $\sigma \subset X$ by least area planes spanning $\Gamma$.

Remark 2.1. Gabai proved this theorem for hyperbolic 3-spaces with cocompact metric in [Ga1].

\section{Properly embedded least area plane}

In this section, we will prove the main theorem of the paper. First, we need a simple topological lemma.

Lemma 3.1. Let $\Sigma$ be a plane in $X$. If $\Sigma$ is closed in $X$, then $\Sigma$ is properly embedded.

Proof. Let $f: \operatorname{Int}\left(D^{2}\right) \rightarrow X$ be a parametrization of $\Sigma$. In other words, $f\left(\operatorname{Int}\left(D^{2}\right)\right)$ $=\Sigma$, and $f$ is an immersion which is a homeomorphism with respect to the topology on $\Sigma$ induced from $X$. Let $K$ be a compact set in $X$. Since $\Sigma$ is closed, $K \cap \Sigma$ is also compact. Then, since $f$ is an embedding, $f^{-1}(K)=f^{-1}(K \cap \Sigma)$ is also compact. So, $\Sigma$ is a properly embedded plane.

By Lemma 4.1 of [Co], any leaf of a $D^{2}$-limit lamination is a least area plane. Now, by the above lemma, if a $D^{2}$-limit lamination $\sigma$ consists of only one plane $\Sigma$, i.e. $\sigma=\Sigma$, then $\Sigma$ must be properly embedded as $\sigma$ is closed by definition. Hence, our aim is to construct a sequence of least area disks in $X$ converging to a $D^{2}$-limit lamination $\sigma$ consisting of only one plane, which will be our desired properly embedded least area plane. The following lemma will be very useful to construct such a sequence of least area disks in $X$.

Lemma 3.2. Let $\Gamma_{1}$ and $\Gamma_{2}$ be two disjoint simple closed curves in $S_{\infty}^{2}(X)$. If $\Sigma_{1}$ and $\Sigma_{2}$ are least area planes in $X$ with $\partial_{\infty} \Sigma_{i}=\Gamma_{i}$, then $\Sigma_{1}$ and $\Sigma_{2}$ are also disjoint.

Proof. Assume that $\Sigma_{1} \cap \Sigma_{2} \neq \emptyset$. So, the intersection between $\Sigma_{1}$ and $\Sigma_{2}$ contains a simple closed curve or infinite line.

Let's assume the intersection contains a simple closed curve $\gamma$. Since $\Sigma_{1}$ and $\Sigma_{2}$ are both minimal, the intersection must be transverse on a subarc of $\gamma$ by maximum principle. Now, $\gamma$ bounds two least area disks, $D_{1}$ and $D_{2}$ in $X$, with $D_{i} \subset \Sigma_{i}$. Now, take a larger subdisk $E_{1}$ of $\Sigma_{1}$ containing $D_{1}$, i.e. $D_{1} \subset E_{1} \subset \Sigma_{1}$. By definition, $E_{1}$ is also a least area disk. Now, modify $E_{1}$ by swapping the disks $D_{1}$ and $D_{2}$. Then, we get a new disk $E_{1}^{\prime}=\left\{E_{1}-D_{1}\right\} \cup D_{2}$. Now, $E_{1}$ and $E_{1}^{\prime}$ have same area, but $E_{1}^{\prime}$ has a folding curve along $\gamma$. By smoothing out this curve as in [MY], we get a disk with smaller area, which contradicts $E_{1}$ being the least area.

If the intersection contains an infinite line $l$, since asymptotic boundaries $\Gamma_{1}$ and $\Gamma_{2}$ are disjoint, $l$ cannot limit on the $S_{\infty}^{2}(X)$. So, $l$ must be in the bounded subset of $X$. If either $\Sigma_{1}$ or $\Sigma_{2}$ is properly embedded, then they cannot contain an infinite line in the intersection. This is because, in that case, the limit point must be in the intersection, and this contradicts the characterization of intersection of minimal surfaces, [HS]. So, $\Sigma_{1}$ and $\Sigma_{2}$ are nonproperly embedded least area planes. Also in this case, the same idea shows that $l$ is a proper line in $\Sigma_{i}$ with the induced path metric.

Let $\left\{D_{i}\right\}$ be a sequence of subdisks in $\Sigma_{1}$ such that $D_{1} \subset D_{2} \subset \ldots \subset D_{n} \subset \ldots$ with $\partial D_{i} \rightarrow \partial_{\infty} \Sigma_{1}$. By [Co], $\left\{D_{i}\right\}$ induces a $D^{2}$-limit lamination $\widehat{\Sigma}_{1}$ with $\partial_{\infty} \widehat{\Sigma}_{1}=\partial_{\infty} \Sigma_{1}$. 
This lamination is not empty since $\Sigma_{1}$ is a leaf in the lamination by construction. Similarly, define $\widehat{\Sigma}_{2}$.

Now, since $\widehat{\Sigma}_{i}$ is closed subset of $X$, the limit set of $l$ must be in $\widehat{\Sigma}_{1} \cap \widehat{\Sigma}_{2}$. We claim that the limit set of $l$ produces a simple closed curve in the intersection. Let $p$ be a limit point of $l$, contained in the leaves $\Sigma_{1}^{\prime}$ and $\Sigma_{2}^{\prime}$ where $\Sigma_{i}^{\prime}$ is a least area plane in $\widehat{\Sigma}_{i}$. By the definition of $D^{2}$-limit lamination, there is a small disk $E$ in $\Sigma_{1}^{\prime}$ containing $p$ such that $E_{i} \rightarrow E$ in $C^{\infty}$ topology where each $E_{i}$ is a small disk in $D_{i} \subset \Sigma_{1}$. Since $p$ is in the limit set of $l$, by the convergence of $\left\{E_{i}\right\}, p$ must be an interior point in the limit set of $l$. This implies that $\Sigma_{1}^{\prime} \cap \Sigma_{2}^{\prime}$ contains a simple closed curve, or a proper line in the induced metric. However, containing an infinite line in the limit implies the existence of monogons in $\Sigma_{1}$, and $\Sigma_{2}$ which contradicts being least area, [HS]. So, this implies the existence of a simple closed curve in the intersection of least area planes $\Sigma_{1}^{\prime}$ and $\Sigma_{2}^{\prime}$. Again, this is a contradiction by the second paragraph of this proof.

Now, we will prove the main theorem.

Theorem 3.3. Let $X$ be a Gromov hyperbolic 3-space with cocompact metric, and $S_{\infty}^{2}(X)$ the sphere at infinity of $X$. Let $\Gamma$ be a given simple closed curve in $S_{\infty}^{2}(X)$. Then, there exists a properly embedded least area plane $\Sigma$ in $X$ with $\partial_{\infty} \Sigma=\Gamma$.

Proof. Let $\Gamma$ be a simple closed curve in $S_{\infty}^{2}(X) . \Gamma$ separates $S_{\infty}^{2}(X)$ into two parts, say $\Omega^{+}$and $\Omega^{-}$, which are open disks. Define sequences of pairwise disjoint simple closed curves $\left\{\Gamma_{i}\right\}$ such that $\Gamma_{i}=\partial E_{i}$, where $E_{i}$ is a closed disk in $\Omega^{+}$for any $i$, and $E_{i} \subset \operatorname{Int}\left(E_{j}\right)$ for any $i<j$. Moreover, $\Omega^{+}=\bigcup_{i} E_{i}$ and $\Gamma_{i} \rightarrow \Gamma$.

By Theorem 2.1, for any $\Gamma_{i} \subset S_{\infty}^{2}(X)$, there exists a least area plane $\Sigma_{i}$ with $\partial_{\infty} \Sigma_{i}=\Gamma_{i}$. Note that these least area planes separate $X$ by their construction in Theorem 2.1. Also, let $p^{+} \in \operatorname{Int}\left(E_{1}\right) \subset \Omega^{+}$and $p^{-} \in \Omega^{-}$be two points belonging to different components of $S_{\infty}^{2}(X)-\Gamma$. Let $\beta$ be an infinite geodesic in $X$ asymptotic to $p^{+}$and $p^{-}$. Since each $\overline{\Sigma_{i}}$ separates $\bar{X}, \beta$ intersects $\Sigma_{i}$ for any $i$. Let $x_{i}$ be a point in $\Sigma_{i} \cap \beta$ for any $i$.

Now, we define the sequence of least area disks. Let $D_{i}$ be a least area disk in $\Sigma_{i}$ containing $x_{i}$ such that $i<d_{X}\left(x_{i}, \partial D_{i}\right)<i+1$ where $d_{X}$ is the extrinsic distance in $X$. We claim that the sequence of least area disks $\left\{D_{i}\right\}$ in $X$ converges to a nonempty lamination $\sigma$. As $\alpha_{i}=\partial D_{i}$ converges to $\Gamma$, by Theorem 2.1, $D_{i}$ converges to a lamination $\sigma$ (possibly empty) by least area planes with $\partial_{\infty} \sigma=\Gamma$. Now, we show that the $\sigma$ is nonempty. Since $p^{+} \in \Omega^{+}$and $p^{-} \in \Omega^{-}$, where $\Omega^{ \pm}$are open disks in $S_{\infty}^{2}(X)$, we can find a sufficiently small $\epsilon$ such that $B_{\epsilon}\left(p^{+}\right) \subset \operatorname{Int}\left(E_{1}\right)$ and $B_{\epsilon}\left(p^{-}\right) \subset \Omega^{-}$. Let $\gamma^{+}=\partial B_{\epsilon}\left(p^{+}\right)$and $\gamma^{-}=\partial B_{\epsilon}\left(p^{-}\right)$. Then, $\gamma^{ \pm}$are two simple closed curves disjoint from $\Gamma$. By Theorem 2.1, there are least area planes $P^{+}$and $P^{-}$in $X$ with $\partial_{\infty} P^{ \pm}=\gamma^{ \pm}$. By Lemma 3.2, $P^{ \pm}$are disjoint from $\Sigma_{i}$ for each $i$. So, the finite segment $\hat{\beta}$ of $\beta$ between $P^{+}$and $P^{-}$contains all intersection points $\Sigma_{i} \cap \beta$ for any $i$. Hence, $\left\{x_{i}\right\} \subset \hat{\beta}$. Since $\hat{\beta}$ is a finite segment, it is compact, and the sequence $\left\{x_{i}\right\}$ has a limit point $x$. This shows that the sequence $\left\{D_{i}\right\}$ has a nonempty limit. So, $\sigma$ is a nonempty lamination by least area planes with $\partial_{\infty} \sigma=\Gamma$ as claimed.

Now, we want to show that $\sigma$ consists of only one least area plane $\mathcal{P}$, i.e. $\sigma=\mathcal{P}$. Assume that $\sigma$ contains more than one least area planes. Recall that $\sigma$ is a collection of disjoint least area planes asymptotic to $\Gamma$, and $\sigma$ is a closed subset of $X$. Note that each plane $\mathcal{P}$ in $\sigma$ separates $X$ by construction and by Theorem 4.3, Step 4 
of [Co] (or Proposition 3.9 Step 3 in [Ga1]). Consider the components of $X-\sigma$. Let $X^{+}$be the component of $X-\sigma$ with $\partial_{\infty} X^{+}=\Omega^{+}$. Note that as $\sigma$ is closed, each component of $X-\sigma$ is open, and so is $X^{+}$. Since for each $i, D_{i} \subset \Sigma_{i}$ and $\partial_{\infty} \Sigma_{i}=\Gamma_{i} \subset \Omega^{+}$, by Lemma 3.2, $D_{i} \subset X^{+}$. Since each plane $\mathcal{P}$ in $\sigma$ separates $X$, there is a plane $\mathcal{P}_{1}$ in $\sigma$ such that $\mathcal{P}_{1}=\partial X^{+}$. Let $\mathcal{P}_{2}$ be another least area plane in $\sigma$. We claim that there cannot be such a plane $\mathcal{P}_{2}$ because of the special properties of the sequence of least area disks $\left\{D_{i}\right\}$. Let $Y^{+}$be the component of $X-\mathcal{P}_{2}$ with $\partial_{\infty} Y^{+}=\Omega^{+}$. Clearly, $X^{+} \subset Y^{+}$. Moreover, since either there exists an open complementary region (a component of $X-\sigma$ ), or there is an open region foliated by least area planes in $\sigma$ between $\mathcal{P}_{1}$ and $\mathcal{P}_{2}$, we have $X^{+} \subsetneq \operatorname{Int}\left(Y^{+}\right)$. This means $\mathcal{P}_{1}$ forms a barrier between the sequence of least area disks $\left\{D_{i}\right\}$ and $\mathcal{P}_{2}$. In other words, the sequence $\left\{D_{i}\right\}$ cannot reach $\mathcal{P}_{2}$, so $\mathcal{P}_{2}$ cannot be in the limit. More precisely, if $q \in \mathcal{P}_{2}$, since $D_{i} \subset X^{+}$for any $i$, and $X^{+} \subsetneq \operatorname{Int}\left(Y^{+}\right)$, there is no sequence $\left\{q_{i}\right\}$ with $q_{i} \in D_{i}$ such that $q_{i} \rightarrow q$. So, $\mathcal{P}_{2}$ cannot be in the limit of $\left\{D_{i}\right\}$. Hence, this shows $\sigma$ consists of only one plane $\mathcal{P}_{1}$, i.e. $\sigma=\mathcal{P}_{1}$.

As $\sigma=\mathcal{P}_{1}$ and $\sigma$ is a lamination, $\mathcal{P}_{1}$ is a closed subset of $X$. By Lemma 3.1, $\mathcal{P}_{1}$ is properly embedded in $X$. This shows $\mathcal{P}_{1}$ is a properly embedded least area plane in $X$ with $\partial_{\infty} \mathcal{P}_{1}=\Gamma$ as desired.

\section{FinAL REMARKS}

In this paper, we showed that for any simple closed curve in asymptotic boundary of a Gromov hyperbolic 3-space with cocompact metric, there exists a properly embedded least area plane in the space spanning the curve. The technique we use here is very general, and can be applied to many similar settings. For example, by using results of Lang in [La], these results can be extended to Gromov hyperbolic Hadamard 3-spaces. Similarly, if we have a positive solution to the asymptotic Plateau problem in a 3 -space $X$, then the methods in this paper can be generalized naturally to this case.

On the other hand, as we point out in the Introduction, while we prove the existence of properly embedded least area planes in hyperbolic 3 -spaces, there is no known nonproperly embedded least area plane in $\mathbb{H}^{3}$. By using the regularity results of Hardt and Lin in [HL], we know that if a simple closed curve in $S_{\infty}^{2}\left(\mathbb{H}^{3}\right)$ is $C^{1}$, then any least area plane asymptotic to the curve is properly embedded. However, this is not yet known for $C^{0}$ simple closed curves in $S_{\infty}^{2}\left(\mathbb{H}^{3}\right)$. Intuitively, being least area and nonproperly embeddedness are contradicting notions. As, nonproper embeddednes must produce monogons, one can get a contradiction by using Hass and Scott's surgery arguments for least area objects in [HS]. Hence, we have the following conjecture.

Conjecture. Let $\Gamma$ be a simple closed curve in $S_{\infty}^{2}\left(\mathbb{H}^{3}\right)$. Then, any least area plane $\Sigma$ in $\mathbb{H}^{3}$ with $\partial_{\infty} \Sigma=\Gamma$ is properly embedded.

\section{ACKNOWLEDGMENTS}

I want to thank David Gabai and Yair Minsky for very helpful conversations. Also, I wish to thank the referee for very critical comments.

\section{REFERENCES}

An. M. Anderson, Complete minimal hypersurfaces in hyperbolic n-manifolds, Comment. Math. Helv. 58 (1983), 264-290. MR705537 (85e:53076) 
BM. M. Bestvina, and G. Mess, The boundary of negatively curved groups, J. Amer. Math. Soc. 4 (1991) 469-481. MR1096169 (93j:20076)

Co. B. Coskunuzer, Uniform 1-cochains and Genuine Laminations, Topology 45 (2006) 751784. MR2236377 (2007c:57028)

HL. R. Hardt and F.H. Lin, Regularity at infinity for absolutely area minimizing hypersurfaces in hyperbolic space, Invent. Math. 88 (1987) 217-224. MR877013 (88m:49033)

HS. J. Hass and P. Scott, The Existence of Least Area Surfaces in 3-manifolds, Trans. AMS 310 (1988) 87-114. MR965747 (90c:53022)

Ga1. D. Gabai, On the geometric and topological rigidity of hyperbolic 3-manifolds, J. Amer. Math. Soc. 10 (1997) 37-74. MR1354958 (97h:57028)

Ga2. D. Gabai, personal communication.

Gr. M. Gromov, Hyperbolic groups, Essays in group theory, Math. Sci. Res. Inst. Publ. 8 Springer (1987) 75-263. MR919829 (89e:20070)

La. U. Lang, Asymptotic Plateau problem in Gromov hyperbolic manifolds, Calc. Var. Partial Differential Equations 16 (2003) 31-46. MR1951491 (2003m:49064)

MY. W. Meeks and S.T. Yau, The classical Plateau problem and the topology of three manifolds, Topology 21 (1982) 409-442. MR670745 (84g:53016)

So1. T. Soma, Existence of least area planes in hyperbolic 3-space with co-compact metric, Topology 43 (2004) 705-716. MR2041639 (2005a:57017)

So2. T. Soma, Least area planes in Gromov hyperbolic 3-spaces with co-compact metric, Geom. Dedicata 112 (2005) 123-128. MR2163893 (2006d:53007)

Department of Mathematics, Koc University, Istanbul, Turkey

E-mail address: bcoskunuzer@ku.edu.tr 